\title{
Programa de Eficiência Energética para uma Unidade de Ensino
}

\author{
Energy Efficiency Program for a Teaching Unit
}

\section{Gabriel Chamie Alves de Souza ${ }^{1}$ (Dhttps://orcid.org/0000-0002-5860-8081}

José Bione de Melo Filho' .. (Dorcid.org/0000-0002-9283-3362

${ }^{1}$ Escola Politécnica de Pernambuco, Universidade de Pernambuco, Recife, Brasil,

E-mail do autor principal: gabchamie@yahoo.com.br

\section{RESUMO}

Este artigo apresenta uma análise de eficiência energética em uma unidade de uma instituição privada do ensino fundamental na cidade do Recife. Nesse estudo de caso, foram utilizadas a metodologia prescritiva do Regulamento Técnico da Qualidade RTQ- C para o nível de Eficiência Energética de Edificações Comerciais, de Serviços e Públicos, envolvendo três parâmetros: o sistema de iluminação artificial, o sistema de climatização artificial do ar e o sistema de aquecimento de piscina. Com o principal propósito de buscar com os dados coletados a melhor eficiência energética na edificação conforme os regulamentos da RTQ-C e de diminuir os custos de energia consumida na unidade predial, encontrando assim uma melhor solução econômica. E através de simulações com o programa EVO ( Dialux), dimensionaremos as iluminâncias dos ambientes e com o programa (PVsyst) dimensionaremos um sistema fotovoltaico que atendam toda a demanda de energia elétrica da escola. Serão realizadas também, uma análise financeira de alguns cenários que gerem uma economia de energia elétrica e consequentemente uma redução nos custos operacionais da escola.

PALAVRAS-CHAVE: RTQ-C; Eficiência Energética; Dialux; PVsyst; Viabilidade Técnica-Economica;

\section{ABSTRACT}

The objective of this article is presents an analysis of energy saving in a private elementary school unit in the city of Recife. In this study, the technical regulations of the RTQ-C for the Energy Efficiency level of Commercial, Services and Public Editions were used, with three parameters: the artificial lighting system, the artificial air conditioning system and the pool heating system. With the purpose of seeking the data needed to obtain energy in order to obtain the required energy level and reduce the energy costs consumed. Through software simulations (Dialux), will be design the ambient and software illuminances (PVsyst) will be design a photovoltaic system that would meet all electrical energy demand of the school. They will be censored, put together some of the plans that generate the saving of electricity and consequently a reduction in the operational costs of the school.

KEY-WORDS: RTQ-C; Energy Efficiency; DiaLux; PVsyst; Economic - Technical Feasibility 


\section{INTRODUÇÃO}

As empresas, em um mercado cada vez mais competitivo, estão à procura de novos conhecimentos de gestão e de novos modelos voltados na eficiência de seus processos, a fim de garantir uma gestão mais eficaz, principalmente nos custos operacionais e no uso dos seus recursos. E a energia elétrica como é um bem imprescindível para o funcionamento de qualquer operação, os seus custos requer uma atenção especial. Com bom diagnóstico nas instalações, 0 gestor pode identificar, quantificar e avaliar as perdas de energia existentes nas instalações, buscando tecnologia mais sustentáveis para o uso da energia elétrica.

Baseado no Regulamento Técnico da Qualidade para o Nível de Eficiência de Edificações Comerciais RTQ-C [1]e através dos Programa de Eficiência Energética em edificações (Procel Edifica) e com o Programa de Etiquetagem Nacional de Conservação de Energia (ENCE) [2], que avaliam as construções das edificações em três sistemas distintos : a envoltória; a iluminação; e o condicionamento de ar. No entanto, neste estudo serão avaliados apenas os sistemas de iluminação e do condicionamento de ar e de aquecimento, além de fazermos uma proposta de substituição de fonte de energia elétrica para alimentar toda a unidade de ensino.

Para o processo de etiquetagem dos ambientes serão utilizados o método prescritivo, que esta baseado no cálculo de uma série de variáveis referentes aos aspectos construtivos da edificação com os valores definido pelas normas a RTQ-C e a ISO/CIE 8995-1 [3]. E cada um dos sistemas avaliados, receberá uma nota de eficiência, variando de $A$ (mais eficiente), B, C, D, E (menos eficiente), conforme descrito no RTQ-C [4] na tabela 1.

Tabela 1: Níveis de Eficiência e seus Equivalentes Numéricos.

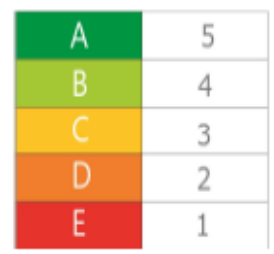

Fonte: RTQ- C (versão 2, p.63)
A classificação final da eficiência da edificação será dada pela soma de todas as avaliações dos equivalentes numéricos dos dois sistemas analisados de acordo com a equação. Será considerado que o sistema de iluminação terá o peso de $30 \%$ e o sistema de condicionamento de ar terá o peso de $40 \%$, conforme a equação 1 [5].

$$
P T=0,30 . \text { EqNumDPI })+0,040\left\{\left(\text { EquumCA. } \frac{A C}{A U}\right)+\left(\frac{A P T}{A U} 5+\frac{A N C}{A U} \text { EqNumV }\right)\right\}+b_{0}^{1}
$$

Onde,

EqNumDPI: equivalente numérico do sistema de iluminação, identificado pela sigla DPI, de Densidade de Potência de Iluminação;

EqNumCA: equivalente numérico do sistema de condicionamento de ar;

EqNumV: equivalente numérico de ambientes não condicionados e/ou ventilados Naturalmente, descrito na tabela 2 .

Tabela 2: Nível de eficiência, definido pelo INMETRO de ar tipo split.

\begin{tabular}{|c|cc|}
\hline Classes & $\begin{array}{c}\text { Coeficiente de eficiência energética (CEE) } \\
\text { Minimo }\end{array}$ & Máximo \\
\hline A & 3.20 & 3.20 \\
\hline B & 3.00 & 3.00 \\
\hline C & 2,80 & 2,80 \\
\hline D & 2,60 & 2,60 \\
\hline E & 2,39 & \\
\hline
\end{tabular}

Fonte: RTQ-C (versão 2, p.182)

APT: área útil dos ambientes de permanência transitória, desde que não condicionados;

ANC: área útil dos ambientes não condicionados de permanência prolongada, com comprovação de percentual de horas ocupadas de conforto por ventilação natural (POC) através do método da simulação;

AC: área útil dos ambientes condicionados;

AU: área útil;

b: pontuação obtida pelas bonificações, que varia de zero a 1.

\section{SISTEMA DE ILUMINAÇÃO}

A luz é a parte integrante no processo de aprendizado escolar. Nas ultimas décadas vem crescendo o número de pesquisas que avaliam as condições de iluminação em edifícios educacionais e que vem demonstrando como a luz influência nos desempenhos das atividades no trabalho, principalmente nas funções motossensorias do 
individuo. Revelando que, a iluminação em uma escola é um fator crítico e com a iluminação deficiente não só é prejudicial ao conforto visual dos ocupantes, mas também pode levar à fadiga visual. Pesquisas mostraram que as fontes de iluminação têm um forte impacto no sistema circadiano (ritmos diários da vigília/sono), onde o espectro da luz do dia é importante para a diminuição da melatonina, que é um hormônio responsável pela regulação do relógio biológico e sua presença facilita o sono; durante o dia, sua concentração no corpo é reduzida, e a luz do dia desempenha um papel muito importante neste processo, figura 1. Estudos revelam que se utilizar um sistema dinâmico de luz artificial, pode estimular mecanismo biológico de maneira a melhorar o estado de atenção e vigilância e sobre a produtividade do individuo. No entanto, as propriedades espectrais do envidraçamento pode levar a exposições inadequadas as certas fontes de luz, alterando o ciclo circadiano do individuo.

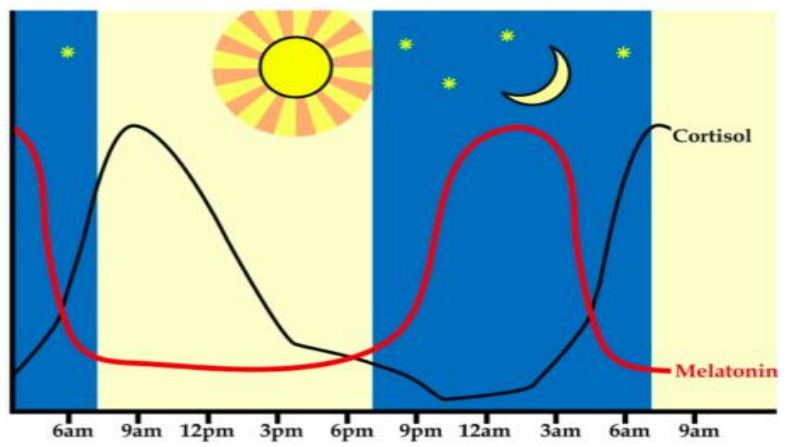

Figura 1: Ciclo Circadiana X Hormônios

Fonte: (https://seucorposeutempo.wordpress.com/2016/11/18/ melatonina-o-hormonio-do-escuro/)

Em base desses argumentos, o Projeto de iluminação School Vision [6], que foi desenvolvida pela Philips e implantada em uma escola básica de Veldvest De Rank em Veldhoven no sul da Holanda em duas turmas, demonstra como é importante a distribuição espectral da luz no ambiente acadêmico. Este sistema inovador, procura criar a atmosfera nas salas de aula ideal para a aprendizagem, permitindo que os docentes escolham entre quatro cenários de iluminação predefinidos: "Normal", "Energia", "Foco" ou "Calmo". Estas definições podem ser utilizadas para acalmar as crianças quando estão muito enérgicas, despertá-las quando estão apáticas ou ajudá-las a concentrar-se durante tarefas complexas. Os resultados preliminares 49 demonstraram resultados bastantes satisfatórios, onde a concentração foi $8,7 \%$ superior na sala de aula com a School Vision. Este valor aumentou para $13,6 \%$ no mês seguinte e espera-se um aumento dos resultados a longo prazo.

\section{SISTEMA DE CONDICIONAMENTO DO AR}

Baseado no programa de etiquetagem da Procel [7] os equipamentos de refrigeração foram classificados individualmente de acordo com os seus selos e foram avaliados também em função da área ocupada conforme os requisitos preconizados pela norma NBR 6401 Instalações centrais de ar-condicionado para conforto Parâmetros básicos de projeto [8]. Com base nas normas vigentes, o dimensionamento de cada equipamento foi considerado para cada metro quadro uma carga estimada de 600 Btu's e a soma de 600 Btu's para cada indivíduo que ocupa a sala.

\section{SISTEMA DE AQUECIMENTO NAS PISCINAS.}

É importante considerar que quanto maior for a diferença de temperatura da superfície da água da piscina e da temperatura do ar imediatamente acima da superfície, maior será a taxa de evaporação da água e, com isso, a taxa de transferência de calor da água para o meio também aumenta consideravelmente, levando a um maior consumo de energia para a recuperação (manutenção) da temperatura da água. Por isso, é necessário fazer uma, instalação de um sistema simples de automação para que o controle de temperatura acione o sistema de aquecimento, composto por um sensor de temperatura, um controlador de temperatura e um relé de comando para a moto bomba do sistema de filtragem e de aquecimento, tornando-o o processo de aquecimento bem mais racional e eficaz, além reduzir consideravelmente os custos com a manutenção do sistema, já que tanto a bomba de calor, não foi concebidos para operar por períodos de tempo muito longos sem interrupções.

E para obter a Classificação A, segundo a RTQC, o sistema tem como pré-requisito geral, ter $100 \%$ da demanda de água quente e se for de classificação B, ter $70 \%$ da demanda de água quente, proveniente de um ou mais dos sistemas 
listados abaixo :

$>$ Sistema de aquecimento solar com os coletores solares voltados para o Norte Geográfico.

$>$ Sistemas de aquecimento de água por bombas de calor ou trocador de calor devem possuir COP igual a 3,0 W/W, conforme descrito na tabela 3.

Tabela 3: Nível de eficiência, definido pelo INMETRO.

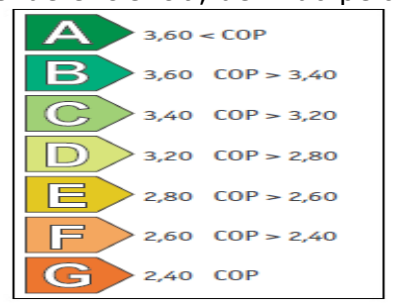

Fonte: Os autores.

\section{ESTUDO DE CASO.}

\subsection{Características do Estabelecimento}

O artigo esta baseado em uma das instalações do Colégio Primeiro Passo que fica localizado no bairro de Boa Viagem da cidade do Recife, atendendo alunos da faixa etária 1,5 ao 10 anos, ocupando uma área de mais $3.000 \mathrm{~m}^{2}$. A unidade predial atende 637 alunos nos dois turnos. Seu espaço físico compreende em dois pavimentos, possuem duas quadras poli esportiva, 30 salas de aulas, dormitório, refeitório, sala de baile, sala de judô, briquedoteca, perfazendo um total de 103 ambientes a serem analisados. Conforme descrito nas plantas baixa das figuras 2 e 3, os ambientes estão mapeados de acordo com as suas principais atividades realizadas em cada ambiente, tabela 4.

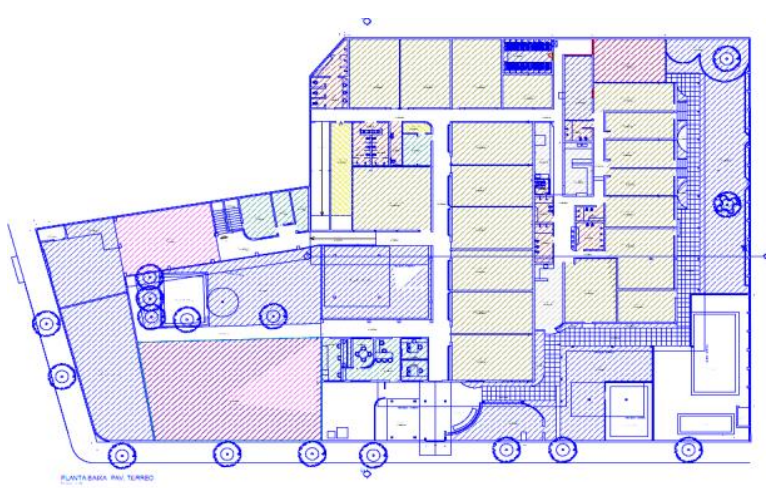

Figura 2: Planta baixa do Térreo com suas principais atividades.

Fonte: Os Autores.
Tabela 4: Descrição dos ambientes em função das

Atividades.

\begin{tabular}{|l|l|}
\hline \multicolumn{1}{|c|}{ Cor } & Ambiente \\
\hline Amarelo & Salas de aula \\
\hline Vermelho & Ginasio,Academia \\
\hline Verde & $\begin{array}{l}\text { Recepção, Secretaria, } \\
\text { Diretoria, Adminsitração }\end{array}$ \\
\hline Branco & Circulação \\
\hline Azul & Refeitorio \\
\hline Cinza & WC, vestuario \\
\hline
\end{tabular}

Fonte: Os Autores.

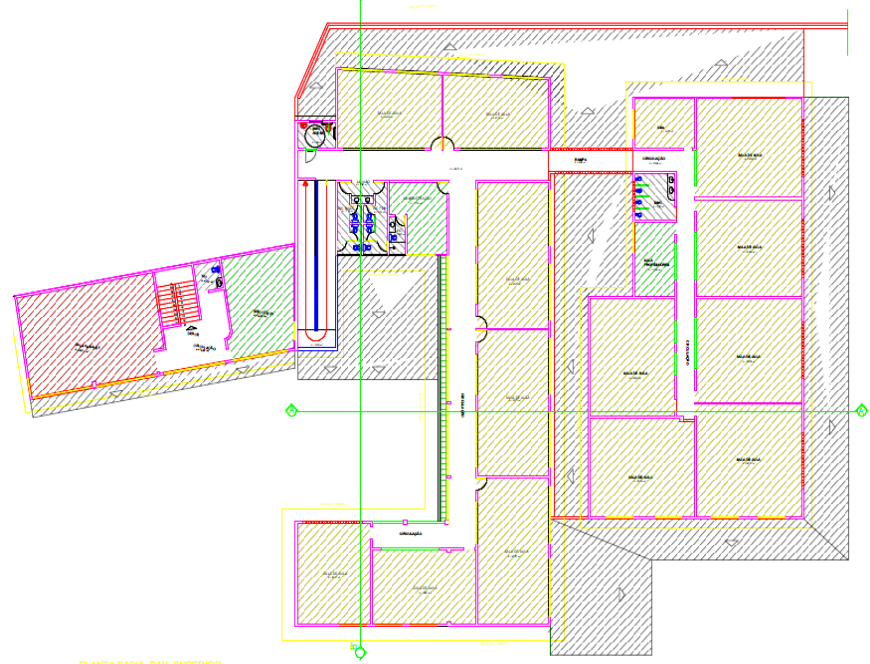

Figura 3: Planta do piso superior com suas principais atividades.

Fonte: Os autores.

\subsection{Sistema de Iluminação}

O sistema de iluminação do Colégio Primeiro Passo utiliza aproximadamente 933 lâmpadas, sendo fluorescente tubular do tipo TL8 de 20W e de 40W, PL 12 W, lâmpadas mistas $250 \mathrm{~W}$ e de vapor de metálico, totalizando uma carga de iluminação estimada de $4.384,72$ W. No qual foram realizados um levantamento quantitativo das lâmpadas e de luminárias e sua disposição no edifício, com suas respectivas potências individuais e totais.

Pontuamos também, algumas características do sistema instalado, como: a distribuição inadequadas (falta de uniformidade) nos níveis de iluminância, luminárias ( vários tipos e com baixo índice de refletância), presença de ofuscamentos dentro do campo de visão, o índice de reprodução de cor no ambiente inapropriado. E para auxiliar na tarefa de encontrar uma melhor forma de padronizar os ambientes e de melhorar os níveis de iluminância, foi utilizada a ferramenta, o 
programa Dialux EVO 8.0, conforme as figuras (4), (5) e (6).

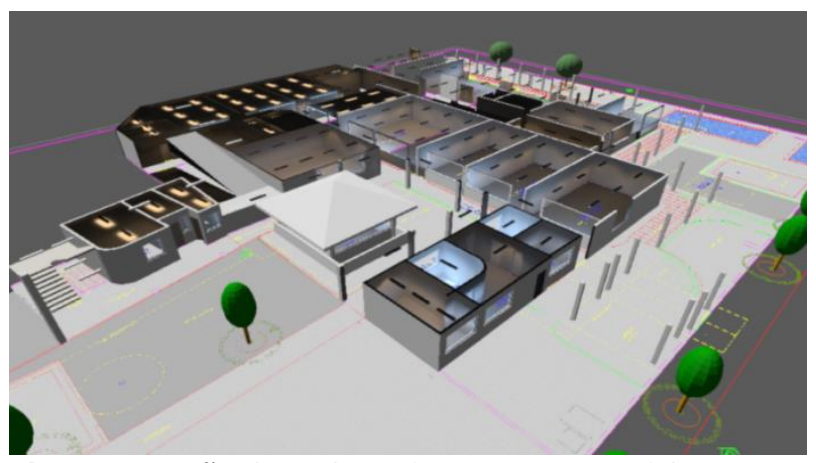

Figura 4: Visão do andar Inferior com cores falsas Fonte: Os Autores.

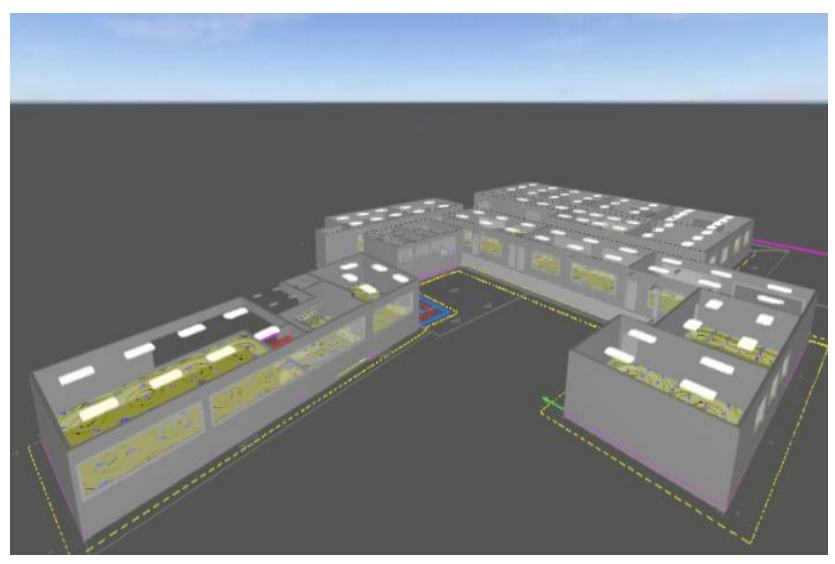

Figura 5: Visão do andar Superior com cores falsas. Fonte: Os Autores.

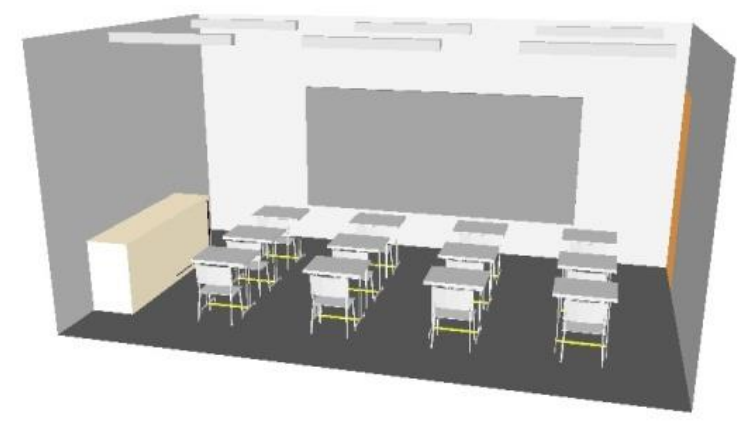

Figura 6: Representação em 3D de uma sala de aula. Fonte: Os autores.

Em conjunto com os resultados obtidos pelo programa Dialux EVO e utilizando o método das atividades, recomendado pelo RTQ-C, que analisa as atividades desenvolvidas para cada ambiente, observou que podemos substituir várias luminárias que apresentam baixo índice de refletância e de luminosidade, conforme a Norma ISO/CIE 8995-1.
A proposta é a substituição de 766 lâmpadas por Led, o que vai apresentar uma redução de consumo de energia de forma bem representativa, além de atender a Densidade de Potencia de Iluminação DPL e os três pré - requisitos estabelecidos pela RTQ-C, que são:

> Divisão de circuitos, cada ambiente fechado deve possuir pelo menos um dispositivo de controle manual para o acionamento independente da iluminação interna do ambiente e cada controle manual deve ser facilmente acessível e controlado, localizado de forma que permita a visão clara de todo ambiente, requisito parcialmente atendido, figura 8;

> Contribuição de luz natural, as fileiras mais próximas as janelas e ou com aberturas ao ambiente externo devem possuir dispositivo de acionamento manual ou automático para o acionamento independente da fileira de luminárias mais próxima à abertura, de forma a propiciar o aproveitamento da luz natural disponível, requisito parcialmente atendido.

> Desligamento automático, como as áreas tem menos de $250 \mathrm{~m}^{2}$, não será necessário o uso de foto sensor.

Dessa forma, conforme RTQ- C da tabela 5 classifica, com o cumprimento parcial dos três prérequisitos específicos e leva a classificação final de nível de eficiência do sistema de iluminação pelo método das atividades para o nível A.

Tabela 5: Pré- requisitos específicos de Iluminação.

\begin{tabular}{|c|c|c|c|}
\hline Prie-Requisito & IViveld & Vivel B & livelC \\
\hline Divisand dos Circuitos & Sill & Siml & $\sin$ \\
\hline 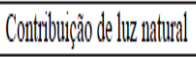 & Sim & Sill & - \\
\hline Desligamentoto automáticico & Sim. & - & - \\
\hline
\end{tabular}

Fonte: RTQ-C.

\subsection{Sistema de Condicionamento de Ar}

Nas instalações do colégio foram verificados que existem um expressivo número de splits $\mathrm{Hi}$ Wall trifásicos da categoria $\mathrm{D}$ e devido os ambientes necessitarem de maiores potencias térmicas, acima de 28.000 Btus e do mercado não oferecer equipamentos individuais com nível de eficiência de nível A e B. 
Dos 30 ambientes avaliados foram encontrados 11 ambientes com equipamentos de eficiência de nível $A$ e $B$, e 19 ambientes com com nível de eficiência $D$ levando o índice global de Coeficiente de Eficiência Energética (CEE)do prédio para 2,73. Foram também, observados que em 14 ambientes apresentaram um baixo nível de carga térmica instalada, conforme a tabela 6, levando o valor do EqNumCA para valores baixo, determinando a classificação final do sistema para nível $D$, influenciando negativamente 0 valor final da pontuação total, por representar um peso de $40 \%$ na classificação final da eficiência na edificação.

Tabela 6: Ambientes fora dos padrões térmicos.

\begin{tabular}{|c|l|c|c|c|c|c|c|c|}
\hline Iten & Ambiente & Área m $^{2}$ & $\begin{array}{c}\text { Ocupação } \\
\text { média } \\
\text { pessoas }\end{array}$ & Aparelhos & $\begin{array}{l}\text { Consumo } \\
\text { de Energia } \\
\text { KW/mês }\end{array}$ & $\begin{array}{l}\text { Potência } \\
\text { Instalada } \\
\text { KBtu's }\end{array}$ & $\begin{array}{l}\text { Categoria } \\
\text { Eficiência }\end{array}$ & $\begin{array}{l}\text { Potencia } \\
\text { Sugerida } \\
\text { KBtu's }\end{array}$ \\
\hline 1 & sala de arte & 61,97 & 20 & 1 & 82,3 & 36 & $\mathrm{D}$ & 49182 \\
\hline 2 & Dept. Esportes & 16,44 & 5 & 1 & 22,8 & 12 & $\mathrm{~A}$ & 12864 \\
\hline 3 & sala 22 & 52,7 & 20 & 1 & 82,3 & 36 & $\mathrm{D}$ & 43620 \\
\hline 4 & sala 24 & 52,7 & 20 & 1 & 82,3 & 36 & $\mathrm{D}$ & 43620 \\
\hline 5 & sala 25 & 52,7 & 15 & 1 & 82,3 & 36 & $\mathrm{D}$ & 40620 \\
\hline 6 & sala 29 & 52,59 & 21 & 1 & 82,3 & 36 & $\mathrm{D}$ & 44154 \\
\hline 7 & sala 30 & 51,88 & 24 & 1 & 82,3 & 36 & $\mathrm{D}$ & 45528 \\
\hline 8 & sala 31 & 55,28 & 21 & 1 & 82,3 & 36 & $\mathrm{D}$ & 45768 \\
\hline 9 & sala 32 & 60,11 & 25 & 1 & 82,3 & 36 & $\mathrm{D}$ & 51066 \\
\hline 10 & sala 34 & 51,84 & 21 & 1 & 82,3 & 36 & $\mathrm{D}$ & 43704 \\
\hline 11 & sala 35 & 50,04 & 20 & 1 & 82,3 & 36 & $\mathrm{D}$ & 42024 \\
\hline 12 & sla. Coordenação & 14,96 & 5 & 1 & 17,1 & 9 & $\mathrm{D}$ & 11976 \\
\hline 13 & biblioteca & 34,33 & 6 & 1 & 36,6 & 18 & $\mathrm{~B}$ & 24198 \\
\hline 14 & s. balet & 69,21 & 15 & 2 & 136,8 & 30 & $\mathrm{D}$ & 50526 \\
\hline
\end{tabular}

Fonte: Os Autores.

\subsection{Sistema de Aquecimento das Piscinas}

Foram escolhidos para a pesquisa, duas piscinas em um mesmo ambiente, sob condições iguais de micro-clima, com um único tipo de sistema de aquecimento, uma bomba de calor elétrica, que esta interligada nas duas piscinas, uma com capacidade de 70 mil litros e a outra com capacidade de 10 mil litros.

No inicio da pesquisa, existia um trocador de calor Jelly modelo BC- 65 [9] que vinha aquecendo as piscinas há mais de quinze anos, com uma capacidade de aquecimento térmico de 64.200 Btu/h e um COP de 6,3 (Coeficiente de Performance), conforme figura 7. O sistema vinha apresentando diversos problemas de vazamentos e elétricos. Inicialmente era TRIFÁSICO $380 \mathrm{~V}$ e devidos há vários consertos, acabaram modificando para MONOFÁSICA 220V, tornando 52 um sistema menos eficiente quanto ao consumo, além do seu sistema de controle de temperatura esta sempre apresentando constantes falhas.

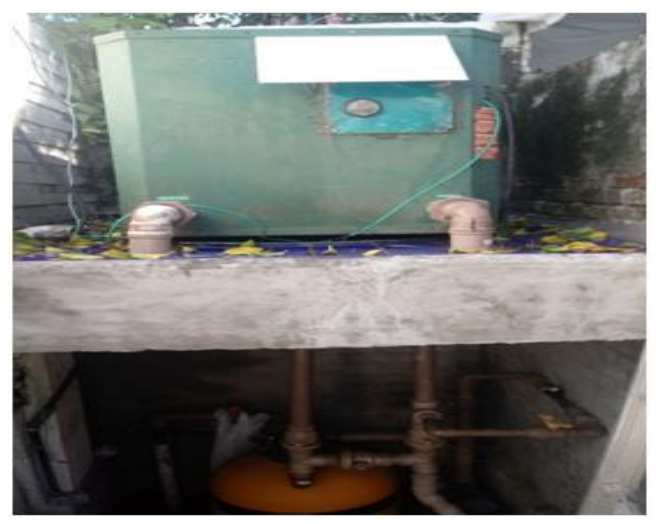

Figura 7: Antiga Instalação do trocado de calor Fonte: Os autores.

A princípio foi realizado um estudo de viabilidade técnica e econômica para implantar um 
sistema de aquecimento térmico solar. Infelizmente, não pode ser aplicável devido a estrutura física do telhado do Colégio não poder abrigar todos os coletores na posição Norte, mas, favorecendo a instalação em outras partes, o que geraria baixa eficiência térmica e um custo elevado de instalação. E devido as constantes quebras foi substituído o trocador de calor por um Sodramar SD 80[10], que tem uma capacidade de aquecimento térmico de 78.54 $\mathrm{Btu} / \mathrm{h}$ e um COP de 5,19 (Coeficiente de Performance), conforme figura 8.

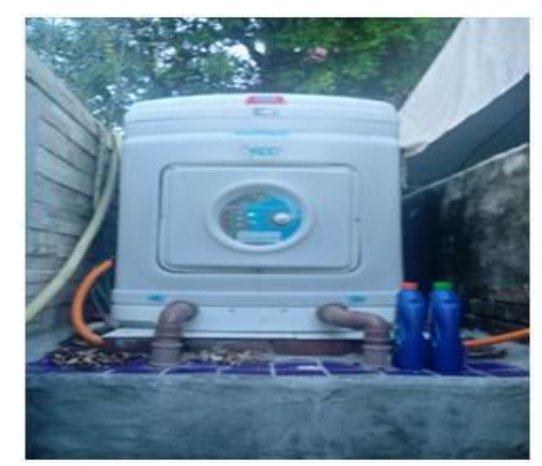

Figura 8: Trocador de Calor Sodramar SD 80 Fonte: Os Autores.

\subsection{Resultados obtidos de acordo com os requisitos da RTQ-C.}

De acordo com a equação 1 apresentada anteriormente na seção 1 , a classificação final da eficiência da edificação, será dada pela soma de todas as avaliações dos equivalentes numéricos dos dois sistemas analisados, de iluminação e de condicionamento do ar, conforme descrito na equação 2 .

$$
\mathrm{PT}=0,30 .(5)+0,40.2\left(\frac{1 \cdot 094,53}{2 \cdot 569,17}\right)=1,84
$$

\section{ESTUDO DE VIABILIDADE ECONÔMICA}

Em função da conta de energia encontrada na unidade consumidora e sob uma análise das tarifas praticadas pela CELPE, tabela 8 , podemos avaliar o desempenho técnico e econômico dos sistemas de (iluminação, refrigeração, aquecimento) antes e após a substituição ou a inserção de componentes que o integram. Permitindo com isso, um estudo mais próximo da realidade. $\mathrm{E}$ através do levantamento de carga realizado em cada sistema, representado na figura 9 , verifica-se o percentual de participação de cada sistema no consumo geral.

Tabela 8: Consumo ativo do Colégio.

\begin{tabular}{|c|c|c|c|c|}
\hline Parcelas Gerenciáveis & Grandezas & Valores unitários & Valores & $\begin{array}{l}\text { Possibilidad } \\
\text { de reduçấo }\end{array}$ \\
\hline Demanda contratada & 97 & 19,3539 & 9 R\$1.877,33 & $x$ \\
\hline Consumo Ativo na ponta(KWh) & 671,89 & 1,98467 & $7 \mathrm{R} \$ 1.333,48$ & $x$ \\
\hline Consumo ativo FORA PONTA(KWh) & $15.621,00$ & 0,36658 & \begin{tabular}{|l|l|}
$8 \$ 5.726,35$ \\
\end{tabular} & $x$ \\
\hline Consumo reativo exec PONTA(KVARh) & 6,05 & 0,331108 & $R \$ 2,00$ & $x$ \\
\hline Consumo reativo exec FORAPONTA(KVARh) & 26,14 & 0,331108 & $\mathrm{R} \$ \$ 8,66$ & $x$ \\
\hline Total de consumo & $16.325,08$ & KWh & R\$\$.947,81 & \\
\hline
\end{tabular}

Fonte: Os Autores.

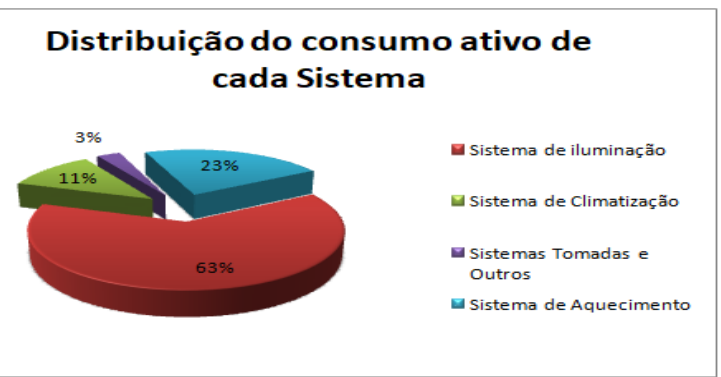

Figura 9: Consumo Ativo de cada sistema Fonte: Os Autores.

Mapeado o perfil de consumo, encontramos o primeiro cenário com diversos tipos de lâmpadas e em alguns ambientes com baixo índice de luminância, visualizado na figura 10 .

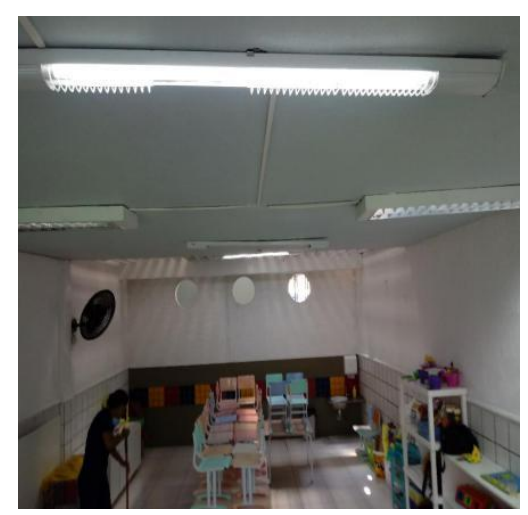

Figura 10: Vários tipos de luminárias no mesmo ambiente.

Fonte: Os Autores.

De posse do resultado obtido anteriormente e sabendo-se que o objetivo deste artigo é classificar o colégio com a melhor nota possível de eficiência, algumas soluções foram preparadas para tentar contornar os problemas encontrados no cenário 1, com a criação de alguns outros cenários, como: 
cenário 2 substituir todas as lâmpadas por Led; cenário 3 substituir a bomba de calor por um sistema mais eficiente e autônomo; cenário 4 implementar um sistema fotovoltaico que atenda a demanda de consumo de energia de toda a escola.

Neste artigo $o$ estudo da viabilidade econômica será realizados para os cenários 2 e 4. A primeira, esta relacionada com a carga do sistema de iluminação que será reduzida em função das trocas de todas TL 18 e $36 \mathrm{w}$ fluorescentes por LED Essential Led Tube da Philips (LEDTUB9W220/86 e LEDTUB18W220/865) [11] que tinha uma carga estimada de $10.194,2 \mathrm{KWh} / \mathrm{mês}$ passa a ter uma carga estimada de 3278,88 KWh/mês, havendo uma redução de $32 \%$.

Foram considerados que o custo médio da energia é de $R \$ 0,43 \mathrm{KW} / \mathrm{h}$, e os investimentos iniciais para a substituição de 14 lâmpadas do tipo (LEDTUB9W220/865) [11] foram estimados em $R \$ 366,80$ e para as 752 lâmpadas do tipo (LEDTUB18W220/865) [11] para valores de $R \$ 24.740,80$. Também foi levado em consideração a economia gerada pela diminuição do consumo.

Foram obtidos um de retorno do investimento deste retrofit a partir do segundo ano para as lâmpadas LEDTUB9W220/865 e no terceiro ano para as lâmpadas LEDTUB18W220/865, conforme representados nas figuras 11 e 12 .

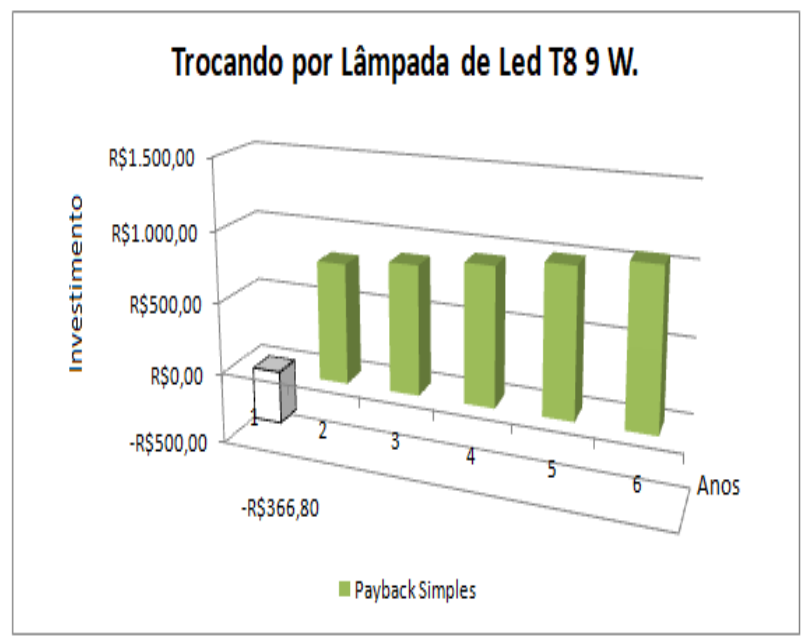

Figura 11: Payback para as LEDTUB 18W220/865 Fonte: Os Autores.

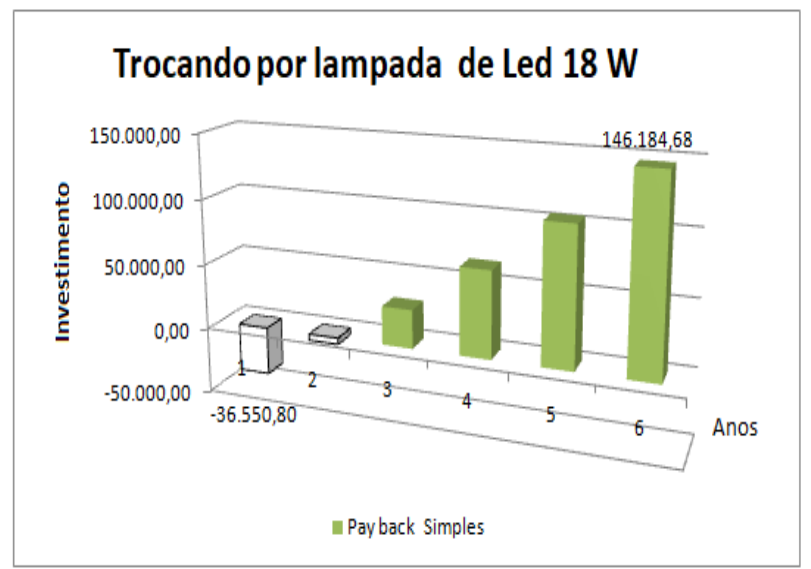

Figura 12: Payback para as LEDTUB9W220/865 Fonte: Os Autores.

Após o retorno deste investimento podemos executar a segunda etapa do estudo, que será a implementação de um sistema que utilize uma fonte de energia renováveis utilizando um sistema fotovoltaico que captará uma a radiação média de $5,3 \mathrm{kWh} / \mathrm{m}^{2}$. dia nos seus painéis de podendo gerar em média $11.500 \mathrm{KWh} /$ mês com potencia nominal de $92,10 \mathrm{KWp}$ numa área $520 \mathrm{~m}^{2}$ disponível no telhado do estacionamento do Colégio, afim de diminuir os custos de energia e que atenda a nova demanda. Serão levados em considerações alguns paramentos, tabela 9.

Tabela 9: Paramentos para o Sistema Solar

\begin{tabular}{|l|c|}
\hline Média HSO Anual: & 5,3 \\
\hline Potência KWp & 93,10 \\
\hline Perdas \% & $17 \%$ \\
\hline
\end{tabular}

Fonte: autor

Com base nos paramentos apresentados anteriormente e utilizando o programa PVsyst 6.7, como uma ferramenta de auxiliar para dimensionar os componentes do sistema fotovoltaico, figura 13. Onde as quantidades e especificações dos equipamentos estão descritos, tabela 10.

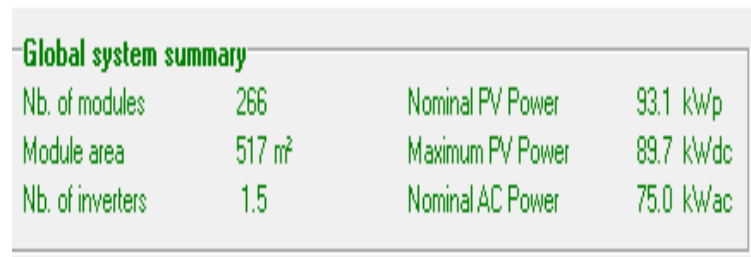

Figura 13: Dados fornecidos pelo PVsyst 6.7. Fonte: Os Autores. 
Tabela 10 - Descrição dos componentes do sistema PV.

\begin{tabular}{|c|c|}
\hline Descrição & Qtde \\
\hline MODULO CANADIAN SOLAR 144 CELLS $355 \mathrm{~W}$ POLY-SI - $1500 \mathrm{~V}$ - Ku & 266 \\
\hline ABB TRIO-TM-5O_-O-AOO-POWER MODULE - 3MPPT - WI-FI INTEGRADO - 38OV & 2 \\
\hline TRANSFORMADOR ISOLADOR Rebaixador 380/220V TRI Ynyno - 100kVA. & 1 \\
\hline SICES SOLAR PERFIL ALUMINIO INDUSTRIAL ROOFTOP 6,3MT & 80 \\
\hline SICES SOLAR PERFIL ALUMINIO INDUSTRIAL ROOFTOP 1.57MT & 26 \\
\hline SICES SOLAR TERMINAL FINAL $39.41 \mathrm{MM}$ fOr CAN - NACIONAL & 56 \\
\hline SICES SOLAR TERMINAL INTERMEDIARIO $39.44 M M$ fO CAN/AVP - NACIONAL & 504 \\
\hline SICES SOLAR PARAFUSO METALICO AUTOPERFURANTE & 2772 \\
\hline$M$ EPDM BAND $30 \times 3, P U=8$ FITA EPDM & 138 \\
\hline CONECTORES FEMEA/ MACHO WEID_CABUR_TE_MC4_ou compativel & 28 \\
\hline CABO SOLAR $6 M M$ ATE $1800 \mathrm{~V}$ CC PT ABNT NBR 16612 & 420 \\
\hline CABO SOLAR GMM ATE $1800 \mathrm{~V}$ CC VM ABNT NBR 16612 & 420 \\
\hline ACESSORIO WALL MOUTING BRACKET - TRIO-50 - ABB & 2 \\
\hline CONVERSOR USB-1485 -NOVUS & 2 \\
\hline ACESSÓRIO DCWB-SX2-TRIO-TM-5O-0-400 & 2 \\
\hline ACESSÓRIO ACWVB-SX2-TRIO-TM-50.0-400 & 2 \\
\hline
\end{tabular}

Fonte: Os autores.

Nesta segunda etapa com instalação de painéis solares e levando em considerações alguns critérios:

\section{Considerações e Critérios.}

1. A produção da planta solar está relacionada à radiação solar mensal da localidade. Como há variação da radiação solar durante os meses do ano, a geração pode variar.

2. No dimensionamento do sistema foram considerados $11,5 \%$ de perdas por temperatura, $1,9 \%$ de perdas na conversão CC-CA, $1,7 \%$ de perdas por sujeira e sombreamento e $1,2 \%$ de perdas no cabeamento, $0,7 \%$ outros totalizando $17 \%$ de perdas.

3. Para estudos solarimétricos do local, uma série de dados de radiação foram importados da base de dados radiométricos METEONORM 7.1, ferramenta de estimação via satélite, onde realizado um estudo da área no período de um ano. Este modelo visa reproduzir séries temporais de irradiação, com comportamento estatístico análogo aos valores medidos em diversas partes do mundo, resultando assim em uma séria temporal horária sintética.

4. Para este sistema que será conectado à rede, a utilização deste método costuma apresentar diferenças na produção energética anual na ordem de 0,5 a $1 \%$.

5. A tarifa terá um ajuste anual de $8 \%$.

6. Na simulação foi considerado a captação do recurso para financiamento dado pelo programa FNESOL do Banco do Nordeste, onde foi considerado com uma taxa de financiamento em $5 \%$ ao ano.

A implantação será dada numa área reservada para estacionamento de pais e professores onde ocupará uma área aproximada $511 \mathrm{~m}^{2}$ de coberta, figura 14 . 


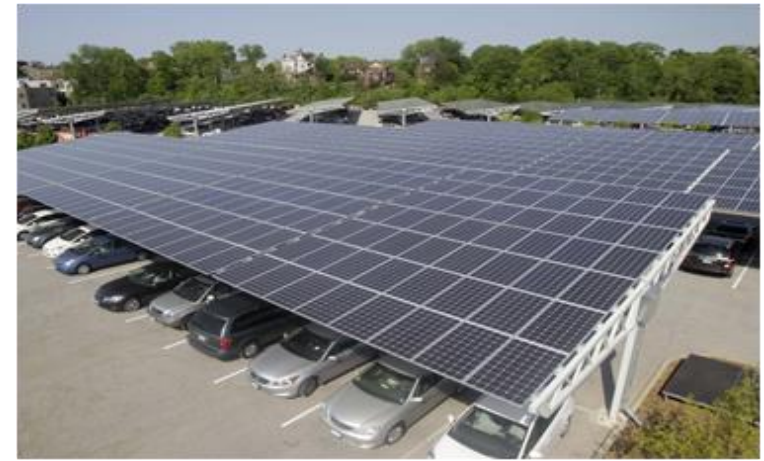

Figura 14: Foto ilustrativa dos painéis solar numa coberta do estacionamento.

Fonte: Solarenergy.

Nesta segunda de etapa de estudo de viabilidade econômica para a implantação de um sistema fotovoltaico, estão sendo considerados que: o custo de instalação é de $\mathrm{R} \$ 4.900,00$ por Kwp, perfazendo um total de $\mathrm{R} \$ 451.230,00$ para toda a unidade de geração; o Colégio fez a opção de ser um consumidor de Tarifa B Optante não pagando mas a demanda contratada, somente a taxa de disponibilidade; por migrar para o uso de um sistema de energia renovável, tem uma isenção de impostos ICMS/PIS/CONFIS sobre a fatura. Levando em conta estas considerações acima citadas, o Colégio após a migração terá uma economia anual aproximada de $\mathrm{R} \$ 107.504,64$ na sua fatura. E com essa economia anual terá como custear esta instalação, obtendo um retorno de investimento a partir do quarto $\mathrm{n}$ ano, figura 15.

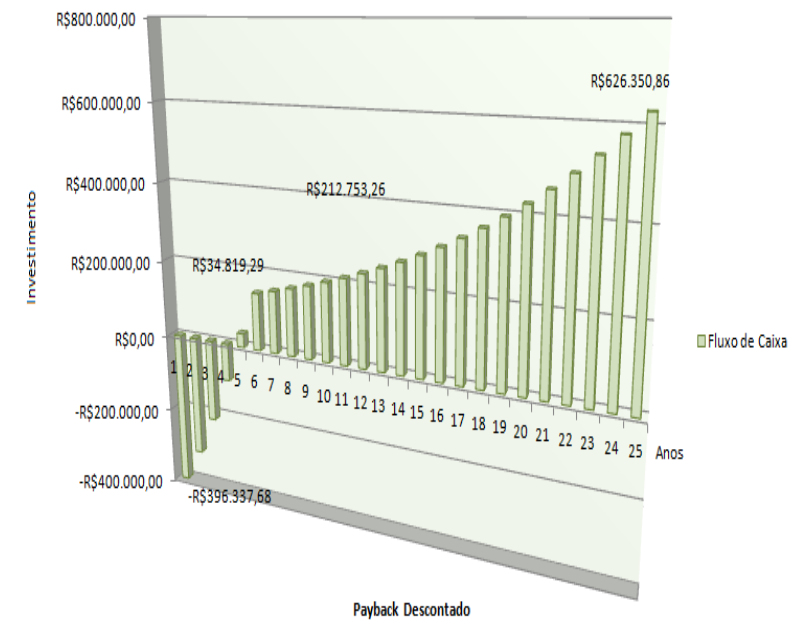

Figura 15: Estudo de viabilidade para a implementação de um sistema PV. Fonte: Os autores.

\section{Conclusões}

Neste artigo foram analisados e aplicado o Regulamento Técnico da Qualidade para o Nível de Eficiência Energética de Edificações Comerciais, de Serviço e Públicas, RTQ-C, regulamento vigente no Brasil, que se refere a eficiência energética em edificações, com o objetivo de tornar o colégio com conceitos ecologicamente correto, buscando identificar ações de cunho sustentável que agreguem ao estabelecimento um maior valor sócio-ambiental, encontrando soluções que ao mesmo tempo preservem os recursos naturais, como também gerem uma economia de energia.

Sabe-se que a tendência é adotar o uso de lâmpadas de Led onde foram obtidos na simulação uma redução de gastos na conta em torno de $34 \%$ e obtendo um nível de eficiência $A$, um resultado bastante satisfatório. Quanto a conservação de energia, deve-se buscar equipamentos que integram ao sistema de refrigeração um CEE/EER ( Coeficiente de Eficiência Energética/Índice de Eficiência Energética) acima de 2,73, conforme a tabela 2. E um COP (Coeficiente de Desempenho) acima de 5 para os equipamentos que integram 0 sistema de aquecimento.

Após realizadas as novas adequações aos sistemas para os padrões estabelecidos de eficiência energética, busca-se uma fonte de energia renovável que atenda a demanda da escola, conforme proposto na simulação de cenário 4, um sistema fotovoltaico on grid.

A grande barreira, sem dúvida, são os elevados investimentos iniciais a realizar, para a substituição de equipamentos mais eficientes, como o sistema e como também, a instalação deum sistema fotovoltaico, onde seus retornos de investimentos terão em media a partir do segundo ano para o sistema de iluminação e para o sistema fotovoltaico a partir do quinto ano.

Infelizmente aPontuação Total para o Nivel de Eficiência do Colégio não ficou satisfatório, nível E, devido a influencia negativa do sistema de condicionamento de ar apresentar um nível de EqNumCA muito baixo, determinado pela classificação final do sistema com nível $D$, conforme o resultado obtido na equação 2 da seção 3.4. 


\section{REFERÊNCIAS}

[1] ASSOCIAÇÃO BRASILEIRA DE NORMAS TÉCNICAS. Regulamento Técnico da Qualidade do Nível de Eficiência Energética para Edificações Comerciais, de Serviço e Públicas (RTQ-C) - Publicado através da portaria $n^{\circ} 372$, de 17 de setembro de 2010.

[2] INMETRO. Requisitos de Avaliação da Conformidade para Eficiência Energética em Edificações (RAC). Publicada através da portaria no 50 de 01 de fevereiro de 2013.

[3] ASSOCIAÇÃO BRASILEIRA DE NORMAS TÉCNICAS. NBR ISO/CIE 8995- 1 Iluminação de ambientes de trabalho. Parte 1: Interior. Rio de Janeiro: ABNT, 2013.

[4] INMETRO. Tabela de edificações comerciais, de serviço e públicas. Disponível em: http://www.inmetro.gov.br/consumidor/pbe/t abelas-comerciais.pdf. Acesso em: $10 \mathrm{dez}$. 2017.

[5] PBE EDIFICA. Manual para Aplicação do RTQ- C. Disponível em: www.pbeedifica.com.br. Acesso em: $10 \mathrm{dez}$. 2017.

[6] PHILIPS. Disponível em: http://www.lighting.philips.com/main/cases/c ases/education/schoolvisionaustria. Acesso em: 12 jan.2018.

[7] PROCEL INFO. Disponível em: www.procelinfo.com.br. Acesso em: $10 \mathrm{dez}$. 2017.

[8] ASSOCIAÇÃO BRASILEIRA DE NORMAS TÉCNICAS. NB 6401: Instalações Centrais de ar-condicionado para conforto - Parâmetros básicos de Projetos. Rio de Janeiro: ABNT, 2008.

[9] Jellyfish -<http://jellyfish.com.br/>.Acesso em 10 dez. 2017.

[10] Sodramar. Disponível em: http://www.sodramar.com.br. Acesso em: 10 dez.2017.

[11] PHILIPS. Guia Prático Philips Iluminação, Lâmpadas, Reatores, Luminárias e Leds 20. Disponível em: http://www.ceap.br/material/MAT25102012 